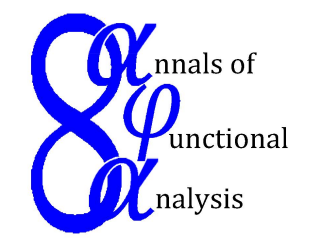

Ann. Funct. Anal. 6 (2015), no. 2, 143-161

http://doi.org/10.15352/afa/06-2-13

ISSN: 2008-8752 (electronic)

http://projecteuclid.org/afa

\title{
HANKEL OPERATORS WITH ANTI-MEROMORPHIC SYMBOLS
}

\author{
E. H. ZEROUALI ${ }^{1 *}$, W. ALHOMSI ${ }^{2}$, H. HACHADI ${ }^{2}$, E. H. YOUSSFI ${ }^{2}$ \\ Communicated by S. R. Garcia
}

\begin{abstract}
We consider Hankel operators $H_{\bar{f}}$ with anti-meromorphic symbols $\bar{f}$ and describe several spectral properties of these operators. Namely, we study their boundedness, compactness and Schatten class membership.
\end{abstract}

\section{INTRODUCTION}

In this paper we consider Hankel operators with anti-meromorphic symbols. The case of anti-holomorphic symbols has a long history. It has been studied by several authors see [1, 3, 5, 6, 29, 34, 35] and [37] in the case of Hardy and Bergman type spaces. The study of Hankel operators in Fock type spaces was considered in the one dimensional setting by [21] and [27] and in the higher dimensional setting by $[15,16,17]$ and $[23]$. The method used by these authors relies on computing the operator norm using the explicit expression for the monomials $z^{k}, k \in \mathbb{N}$. Later, in a joint work of the third named author with Bommier-Hato and $([7,8])$ and with Seip [28], this result was generalized in multi-dimensional case in connection with the Stieltjes moment problem.

We recall that studying the spectral properties of Hankel operators in the context of Bergman spaces and generalized Fock spaces consists in characterizing those symbols $f$ for which the Hankel operator $H_{\bar{f}}$ is bounded (resp. compact, in Schatten class). See Theorem A in [7].

We are interested by study the possibility to have bounded Hankel operators (resp. compact, in Schatten class) whose symbols are not necessarily entire

Date: Received: Jul. 7, 2014; Accepted: Jul. 24, 2014.

${ }^{2}$ Corresponding author.

2010 Mathematics Subject Classification. Primary 47B35. Secondary 47B10.

Key words and phrases. Hankel operators, Hilbert spaces, strong moment problem, Laurent polynomials. 
functions and in particular not polynomials. To this end we consider the space $\mathcal{A}^{2}\left(\mu_{m}\right), m>0$ of all holomorphic functions in $\mathbb{C} \backslash\{0\}$ such that

$$
\int_{\mathbb{C}^{*}}|f(z)|^{2} e^{-\left(|z|^{m}+\frac{1}{|z|^{m}}\right)} d A(z)<+\infty
$$

where $d \mu_{m}(z):=e^{-\left(|z|^{m}+\frac{1}{|z|^{m}}\right)} d A(z)$, and $d A$ is the Lebesgue measure in the complex plane.

The sequence of moments of the measure $\mu_{m}$ are well-defined and given by

$$
s_{d}=\int_{0}^{+\infty} t^{d} e^{-\left(t^{m}+\frac{1}{t^{m}}\right)} d t .
$$

for arbitrary integers $d$ in the set of all integers $\mathbb{Z}$. The notion of Laurent polynomial and related $L$-degree will be introduced in Section 2 and will be used to define Hankel operators. We shall prove that the space $\mathcal{A}^{2}\left(\mu_{m}\right)$ is closed in $L^{2}\left(\mu_{m}\right)$ and denote by $K_{m}(\cdot, \cdot)$ its reproducing kernel. For $f \in \mathcal{A}^{2}\left(\mu_{m}\right)$ the Hankel operator $H_{\bar{f}}$ is defined for symbols in the class $\chi(m)$ by

$$
H_{\bar{f}}(\varphi)(z)=\int_{\mathbb{C}^{*}} K_{m}(z, \omega) \varphi(\omega)[\bar{f}(z)-\bar{f}(\omega)] d \mu_{m}(\omega)
$$

where $\chi(m)$ is the class of functions $f \in \mathcal{A}^{2}\left(\mu_{m}\right)$ such that $f \varphi K_{m}(z,.) \in L^{1}\left(\mu_{m}\right)$ and $\varphi$ is holomorphic Laurent polynomial. Finally, if $f$ is a function defined on $\mathbb{C} \backslash\{0\}$, we set

$$
U f(z)=f\left(\frac{1}{z}\right), z \in \mathbb{C} \backslash\{0\} .
$$

Our results are the following :

Theorem 1.1. Suppose that $f \in \mathcal{A}^{2}\left(\mu_{m}\right)$, where $m>0$. Then

1) The operators $H_{\bar{f}}$ and $H_{\overline{U f}}$ are bounded on the space $\mathcal{A}^{2}\left(\mu_{m}\right)$ if and only if $f$ is a Laurent polynomial of L-degree at most $2 \mathrm{~m}$.

2) The operators $H_{\bar{f}}$ and $H_{\overline{U f}}$ are compacts on the space $\mathcal{A}^{2}\left(\mu_{m}\right)$ if and only if $f$ is a Laurent polynomial of L-degree smaller than $2 \mathrm{~m}$.

3) The operators $H_{\bar{f}}$ and $H_{\overline{U f}}$ are in Schatten class $\mathcal{S}_{p}\left(\mathcal{A}^{2}\left(\mu_{m}\right), L^{2}\left(\mu_{m}\right)\right)$ if and only if $p>2$ and $f$ is a Laurent polynomial of L-degree smaller than $\frac{2 m(p-2)}{p}$.

\section{PRELIMINARIES}

A distribution $d \psi(t)$ is said to be a strong distribution on $(a, b) \subset(0,+\infty)$ if $\psi(t)$ is real-valued, bounded, non-decreasing function and has infinitely many points of increase on $(a, b)$. In this case all the moments

$$
s_{k}=\int_{a}^{b} t^{k} d \psi(t), k \in \mathbb{Z}
$$

exist.

Such distributions are called strong Stieltjes distributions. They were introduced in 1982 in the context of the study of the strong moment problem [19] that 
is, given a sequence of real numbers $\left\{s_{k}\right\}_{-\infty}^{+\infty}$, what are necessary and sufficient conditions about the function $\psi(t)$ on $(0,+\infty)$, such that $(2.1)$ holds. The theory of orthogonal Laurent polynomials appeared later in connection with different type of strong moment problems [10, 18, 20] and [26]. Other extensions of the moment problem have been developed in the context of this theory, especially in the case of rapidly decreasing functions[13].

Herein, we consider Hankel operators in a Hilbert space of meromorphic functions in connection with the strong moment problem. More precisely if the sequence $s=\left(s_{p}\right)_{p \in \mathbb{Z}}$ of the form

$$
s_{d}=\int_{a}^{b} t^{d} d \mu(t) \text { and } s_{-d}=\int_{a}^{b} t^{-d} d \mu(t)=\int_{\frac{1}{b}}^{\frac{1}{a}} t^{d} d \widetilde{\mu}(t)=\widetilde{s}_{d}
$$

where $d \in \mathbb{N}$ and $0 \leq a<b \leq+\infty$. And, furthermore, the measure $\mu$ is a nonnegative measure on $[a, b]$, called a representing measure for $s$, and the measure $\widetilde{\mu}$ is the image measure of $\mu$ under the transformation $t \rightarrow \frac{1}{t}$. We denote by $\mathcal{S}$ the set of such sequences and by $\mathcal{S}^{*}$ the set of all non-vanishing elements of $\mathcal{S}$.

Let $s \in \mathcal{S}^{*}$, by Cauchy-Schwarz inequality we see that the sequence $\left(\frac{s_{d+1}}{s_{d}}\right)_{d \geq 0}$ is non-decreasing and hence converges as $d \rightarrow+\infty$ to the radius of convergence $r_{+}$of the entire series :

$$
F_{+}(\lambda)=\sum_{d=0}^{+\infty} \frac{\lambda^{d}}{s_{d}}, \lambda \in \mathbb{C} .
$$

Similarly, the sequence $\left(\frac{\widetilde{s}_{d+1}}{\widetilde{s}_{d}}\right)_{d \geq 0}$ is non-decreasing and hence converges as $d \rightarrow$ $+\infty$ to the radius of convergence $r_{-}$of the entire series :

$$
F_{-}(\lambda)=\sum_{d=1}^{+\infty} \frac{\lambda^{d}}{\widetilde{s}_{d}}, \lambda \in \mathbb{C}
$$

We set

$$
\begin{gathered}
R_{+}:=\lim _{d \rightarrow+\infty} \sqrt{\frac{s_{d+1}}{s_{d}}}=\sqrt{\lim _{d \rightarrow+\infty} s_{d}^{\frac{1}{d}}} \\
\frac{1}{R_{-}}:=\lim _{d \rightarrow+\infty} \sqrt{\frac{\widetilde{s}_{d+1}}{\widetilde{s}_{d}}}=\sqrt{\lim _{d \rightarrow+\infty} \widetilde{s}_{d}^{\frac{1}{d}}}
\end{gathered}
$$

and assume that $r_{-}<r_{+}$and we define the series

$$
F_{s}(\lambda):=F_{+}(\lambda)+F_{-}\left(\frac{1}{\lambda}\right), r_{-}^{-1}<|\lambda|<r_{+} .
$$

Denote by $\Omega_{s}=\left\{z \in \mathbb{C} / R_{-}<|z|<R_{+}\right\}$the annulus of radius $R_{-}$and $R_{+}$. We denote by $\mathcal{A}^{2}(s)$ the Hilbert space of holomorphic functions $f(z)=\sum_{k \in \mathbb{Z}} a_{k} z^{k}$ on $\Omega_{s}$ that satisfy

$$
\sum_{-\infty}^{+\infty} s_{k}\left|a_{k}\right|^{2}<+\infty
$$


equipped with the standard inner product

$$
\langle f, g\rangle:=\sum_{k \in \mathbb{Z}} s_{k} a_{k} \overline{b_{k}}
$$

if $f(z)=\sum_{k \in \mathbb{Z}} a_{k} z^{k}$ and $g(z)=\sum_{k \in \mathbb{Z}} b_{k} z^{k}$ are two elements of $\mathcal{A}^{2}(s)$.

Now let $\sigma$ be the rotation invariant probability measure on the unit circle and $\mu$ be the representing measure of $s$. We denote by $\mu_{s}$ the image measure on $\mathbb{C}$, of $\mu \otimes \sigma$ under the map $(t, \xi) \rightarrow \sqrt{t} \xi$ from $[a, b] \times[0,2 \pi]$ onto $\mathbb{C}$.

Let $L^{2}\left(\mu_{s}\right)$ be the Hilbert space of all square integrable complex-valued functions with respect to the measure $\mu_{s}$ equipped with the norm

$$
\|f\|_{L^{2}\left(\mu_{s}\right)}^{2}:=\int_{\mathbb{C}}|f(z)|^{2} d \mu_{s}(z) .
$$

In addition, we consider the set of Laurent polynomials (L-polynomials) denoted by $\Lambda$. This is the space of those rational fractions of the form $P(z)=\sum_{j=m}^{n} a_{j} z^{j}$, where $m, n \in \mathbb{Z} m \leq n$ and $a_{j} \in \mathbb{C}$. We denote by $\Lambda_{m, n}$ the set of Laurent polynomials belonging to $\operatorname{span}\left\{z^{j} / m \leq j \leq n\right\}$. The next two classes of Laurent polynomials are of particular interest.

$$
\Lambda_{2 n}=\left\{P \in \Lambda_{-n, n} \text { such that the coefficient of } z^{n} \text { is nonzero }\right\}
$$

and

$$
\Lambda_{2 n+1}=\left\{P \in \Lambda_{-n-1, n} \text { such that the coefficient of } z^{-n-1} \text { is nonzero }\right\}
$$

We define the following inner product on $\Lambda$ by

$$
\langle f, g\rangle=\int_{\Omega_{s}} f(z) \overline{g(z)} d \mu_{s}, \forall f, g \in \Lambda .
$$

For each $P \in \Lambda$, it is obvious that there exists a unique $k \in \mathbb{N}$ such that $P \in \Lambda_{k}$. In this case $P$ is said to be of L-degree $k$. In particular, for the $L$-degree of an ordinary polynomial is twice its degree.

The study of these polynomials first appeared in connection with the strong moments problem [19], and it will be developed later see [20] and [26], the theory of Laurent polynomials extends now to other fields of applications, including quadrature formulas [9] and pade approximation [12] and [22], for more details on the theory of Laurent polynomials see [10]. Another result of this paper is the following

Theorem 2.1. The measure $\mu_{s}$ is supported by the closure of the domain $\bar{\Omega}_{s}$. In addition, for each set compact $K \subset \Omega_{s}$, there exists $M=M(K)>0$ such that :

$$
\sup _{z \in K}|f(z)| \leq M\|f\|_{L^{2}\left(\mu_{s}\right)}
$$

for all holomorphic Laurent polynomials $f$ in $\Omega_{s}$. Furthermore, the space $\mathcal{A}^{2}(s)$ coincides with the closure of the holomorphic Laurent polynomials in $L^{2}\left(\mu_{s}\right)$ and its reproducing kernel is given by

$$
K_{s}(z, \omega)=F_{s}(z \bar{\omega}) z, \omega \in \Omega_{s} .
$$


Proof. Suppose that $r$ is a positif real number that satisfies $r>a$ and $\mu(] r, b[)=0$.

Then for $d \in \mathbb{N}$, we have $s_{d} \leq r^{d} \mu(] a, b[)$ and hence $\lim \sup _{d} s_{d}^{\frac{1}{d}} \leq r$, showing that the radius of convergence $r_{+}$of the series $F_{+}$satisfies

$$
r_{+} \leq \inf \{r: \mu(] r, b[)=0\} .
$$

Conversely, suppose that $r>a$ satisfies $\mu(] r, b[)>0$. Then

$$
r^{d} \mu(] r, b[) \leq s_{d}
$$

Therefore

$$
r \leq \liminf _{d} s_{d}^{\frac{1}{d}} \leq \limsup _{d} s_{d}^{\frac{1}{d}}
$$

since

$$
\inf \{r: \mu(] r, b[)=0\}=\sup \{r: \mu(] r, b[)>0\},
$$

we see that $r_{+}=R_{+}^{2}=\lim _{d \rightarrow+\infty} s_{d}^{\frac{1}{d}}$.

Applying the same arguments to the sequence $\widetilde{s}_{d}$ we show that

$$
r_{-}=R_{-}^{-2}=\lim _{d \rightarrow+\infty} \widetilde{s}_{d}^{\frac{1}{d}}
$$

Thus, the measure $\mu_{s}$ is supported by the closure $\bar{\Omega}_{s}$ and for each $z \in \Omega_{s}$, the series

$$
K_{s}(z, \omega)=\sum_{-\infty}^{+\infty} \frac{1}{s_{d}}(z \bar{\omega})^{d}, \omega \in \Omega_{s}
$$

converges on $\bar{\Omega}_{s}$. Moreover, using the orthogonality of Laurent polynomials with respect to $\mu_{s}$ it follows that

$$
\begin{aligned}
\int_{\bar{\Omega}_{s}}\left|K_{s}(z, \omega)\right|^{2} d \mu_{s}(\omega) & \leq \liminf _{N \rightarrow+\infty} \int_{\bar{\Omega}_{s}}\left|\sum_{-N}^{N} \frac{1}{s_{d}}(z \bar{\omega})^{d}\right|^{2} d \mu_{s}(\omega) \\
& \leq \liminf _{N \rightarrow+\infty} \int_{\bar{\Omega}_{s}} \sum_{-N}^{N}\left|\frac{1}{s_{d}}(z \bar{\omega})^{d}\right|^{2} d \mu_{s}(\omega) \\
& \leq K_{s}(z, z)
\end{aligned}
$$

Hence for any fixed $z$ on $\Omega_{s}$, the series $K_{s}(z, \omega)$ converges in $L^{2}\left(\mu_{s}\right)$, and for $k \in \mathbb{Z}$, we have

$$
\int_{\bar{\Omega}_{s}} \omega^{k} K_{s}(z, \omega) d \mu_{s}(\omega)=\sum_{-\infty}^{+\infty} \frac{1}{s_{d}} \int_{\bar{\Omega}_{s}} \omega^{k}(z \bar{\omega})^{d} d \mu_{s}(\omega)=z^{k} .
$$

This shows that the kernel $K_{s}(z, \omega)$ reproduces holomorphic Laurent polynomials and

$$
\sup _{z \in K}|f(z)| \leq \sqrt{K_{s}(z, z)}\|f\|_{L^{2}\left(\mu_{s}\right)}
$$

for all holomorphic Laurent polynomial $f$ and each compact $K \subset \Omega_{s}$. 


\section{HANKEL OPERATORS}

As mentioned before, the orthogonal projection associated to $\mathcal{A}^{2}(s)$ is given for all $g \in L^{2}\left(\mu_{s}\right)$ by

$$
\left(P_{\mu_{s}} g\right)(z)=\int_{\bar{\Omega}_{s}} K_{s}(z, \omega) g(\omega) d \mu_{s}(\omega), z \in \Omega_{s}
$$

This integral operator can be extended in a natural way to functions $g$ such that $K_{s}(z,). g \in L^{1}\left(\mu_{s}\right)$ for all $z \in \Omega_{s}$. This allows us to define Hankel operators. Indeed, we denote by $\chi(s)$ the class of functions $f \in \mathcal{A}^{2}(s)$ such that $f \varphi K_{s}(z,.) \in$ $L^{1}\left(\mu_{s}\right)$ for each holomorphic Laurent polynomial $\varphi$ and $z \in \Omega_{s}$, and

$$
H_{\bar{f}}(\varphi)(z)=\int_{\bar{\Omega}_{s}} K_{s}(z, \omega) \varphi(\omega)[\bar{f}(z)-\bar{f}(\omega)] d \mu_{s}(\omega), z \in \Omega_{s}
$$

is a densely defined operator from $\mathcal{A}^{2}(s)$ into $L^{2}\left(\mu_{s}\right)$. This operator will be called the Hankel operator with symbol $\bar{f}$ and it can be written in the form

$$
H_{\bar{f}}(\varphi)=\left(I-P_{\mu_{s}}\right)(\bar{f} \varphi)
$$

for all holomorphic Laurent polynomial $\varphi$. For $f \in \chi(s)$, we denote by $\operatorname{spec}(f)$ the set of all indices $k \in \mathbb{Z}$ such that the coefficient of the Laurent series of $f$ is nonzero. It is not hard to see that the class $\chi(s)$ contains all holomorphic Laurent polynomials.

In this section we give the expression of the operators $H_{\bar{z}^{k}}$ and $H_{\bar{z}^{k}}^{*} H_{\bar{z}^{k}}$ for holomorphic Laurent monomials.

Proposition 3.1. Suppose that $k, l \in \mathbb{Z}$, and $g$ is the monomial $g(z)=z^{l}$. Then :

if $k \geq 0$

if $k<0$

$$
P_{\mu_{s}}\left(\bar{z}^{k} g\right)=\left\{\begin{array}{cl}
\frac{s_{l}}{s_{l-k}} \frac{(l-k) !}{l !} \frac{\partial^{k}}{\partial z^{k}} g & \text { if } l \geq k \\
(-1)^{k} \frac{s_{l}}{s_{l-k}} \frac{(-l-1) !}{(-l+k-1) !} \frac{\partial^{k}}{\partial z^{k}} g & \text { if } l<0
\end{array}\right.
$$

$$
P_{\mu_{s}}\left(\bar{z}^{k} g\right)=\left\{\begin{array}{cc}
\frac{s_{l}}{s_{l-k}} \frac{(-l+k) !}{(-l) !} U \frac{\partial^{-k}}{\partial z^{-k}} U g & \text { if } l \leq k \\
(-1)^{k} \frac{s_{l}}{s_{l-k}} \frac{(l-1) !}{(l-k-1) !} U \frac{\partial^{-k}}{\partial z^{-k}} U g & \text { if } l>0
\end{array}\right.
$$

where $U$ is the transformation defined by $U g(z)=g\left(\frac{1}{z}\right)$.

For general Laurent polynomials $g$ the projection can be computed by linearity as $P_{\mu_{s}}\left(\bar{z}^{k} g\right)=\sum_{-p}^{q} \widehat{g}(l) P_{\mu_{s}}\left(\bar{z}^{k} z^{l}\right)$.

Proof. Let $h$ be a holomorphic Laurent polynomial. Using the properties of $P_{\mu_{s}}$, we see that

$$
\left\langle P_{\mu_{s}}\left(\bar{z}^{k} g\right), h\right\rangle_{L_{\left(\mu_{s}\right)}^{2}}=\left\langle g, z^{k} h\right\rangle_{L_{\left(\mu_{s}\right)}^{2}}
$$

In patricular, if $h(z)=z^{m}$ with $m \in \mathbb{Z}$, we have

$$
\left\langle P_{\mu_{s}}\left(\bar{z}^{k} g\right), h\right\rangle_{L_{\left(\mu_{s}\right)}^{2}}=0
$$


as long as $l \neq k+m$. However, when $l=k+m$, we consider the following two cases :

Case where $k \geq 0$

If $l \geq 0$, then

$$
\left\langle P_{\mu_{s}}\left(\bar{z}^{k} g\right), h\right\rangle_{L_{\left(\mu_{s}\right)}^{2}}=\frac{s_{l}}{s_{l-k}} \frac{(l-k) !}{l !}\left\langle\frac{\partial^{k}}{\partial z^{k}} g, h\right\rangle_{L_{\left(\mu_{s}\right)}^{2}}
$$

If $l<0$, then using the observation that

$$
\frac{\partial^{k}}{\partial z^{k}} z^{l}=(-1)^{k} \frac{(-l+k-1) !}{(-l-1) !} z^{l-k}
$$

we get

$$
\left\langle P_{\mu_{s}}\left(\bar{z}^{k} g\right), h\right\rangle_{L_{\left(\mu_{s}\right)}^{2}}=(-1)^{k} \frac{(-l-1) !}{(-l+k-1) !} \frac{\widetilde{s}_{-l}}{\widetilde{s}_{-l+k}}\left\langle\frac{\partial^{k}}{\partial z^{k}} g, h\right\rangle_{L_{\left(\mu_{s}\right)}^{2}} .
$$

Therefore, for $g=z^{l}, l \in \mathbb{Z}$ and $k \geq 0$ we have

$$
P_{\mu_{s}}\left(\bar{z}^{k} g\right)=\left\{\begin{array}{cc}
\frac{s_{l}}{s_{l-k}} \frac{(l-k) !}{l !} \frac{\partial^{k}}{\partial z^{k}} g & \text { if } l \geq k \\
(-1)^{k} \frac{(-l-1) !}{(-l+k-1) !} \frac{\widetilde{s}_{-l}}{\widetilde{s}_{-l+k}} \frac{\partial^{k}}{\partial z^{k}} g & \text { if } l<0
\end{array}\right.
$$

Since $\widetilde{s}_{-l}=s_{l}$, it follows that

$$
P_{\mu_{s}}\left(\bar{z}^{k} g\right)=\left\{\begin{array}{cl}
\frac{s_{l}}{s_{l-k}} \frac{(l-k) !}{l !} \frac{\partial^{k}}{\partial z^{k}} g & \text { if } l \geq k \\
(-1)^{k} \frac{s_{l}}{s_{l-k}} \frac{(-l-1) !}{(-l+k-1) !} \frac{\partial^{k}}{\partial z^{k}} g & \text { if } l<0
\end{array}\right.
$$

Case where $k<0$ We make use of the transformation $U f(z)=f\left(\frac{1}{z}\right)$.

If $l \leq 0$, then

$$
\left\langle P_{\mu_{s}}\left(\bar{z}^{k} g\right), h\right\rangle_{L_{\left(\mu_{s}\right)}^{2}}=\left\langle g, z^{k} h\right\rangle_{L_{\left(\mu_{s}\right)}^{2}}=\left\langle U g, z^{-k} U h\right\rangle_{L_{\left(\tilde{\mu}_{s}\right)}^{2}}
$$

and hence

$$
\left\langle P_{\mu_{s}}\left(\bar{z}^{k} g\right), h\right\rangle_{L_{\left(\mu_{s}\right)}^{2}}=\frac{\widetilde{s}_{-l}}{\widetilde{s}_{-l+k}} \frac{(-l+k) !}{(-l) !}\left\langle U \frac{\partial^{-k}}{\partial z^{-k}} U g, h\right\rangle_{L_{\left(\mu_{s}\right)}^{2}} .
$$

For $l>0$, using the fact that

$$
\frac{\partial^{-k}}{\partial z^{-k}} z^{-l}=(-1)^{k} \frac{(l-k-1) !}{(l-1) !} z^{-l+k}
$$

we get

$$
\left\langle P_{\mu_{s}}\left(\bar{z}^{k} g\right), h\right\rangle_{L_{\left(\mu_{s}\right)}^{2}}=(-1)^{k} \frac{s_{l}}{s_{l-k}} \frac{(l-1) !}{(l-k-1) !}\left\langle\frac{\partial^{-k}}{\partial z^{-k}} U g, U h\right\rangle_{L_{\left(\tilde{\mu}_{s}\right)}^{2}} .
$$

Therefore, for $g=z^{l}, l \in \mathbb{Z}$ and $k<0$ we have

$$
P_{\mu_{s}}\left(\bar{z}^{k} g\right)= \begin{cases}\frac{\widetilde{s}_{-l}}{\widetilde{s}_{-l+k}} \frac{(-l+k) !}{(-l) !} U \frac{\partial^{-k}}{\partial z^{-k}} U g & \text { if } l \leq k \\ (-1)^{k} \frac{s_{l}}{s_{l-k}} \frac{(l-1) !}{(l-k-1) !} U \frac{\partial^{-k}}{\partial z^{-k}} U g & \text { if } l>0\end{cases}
$$


Using again the fact that $\widetilde{s}_{-l}=s_{l}$, wee see that

$$
P_{\mu_{s}}\left(\bar{z}^{k} g\right)=\left\{\begin{array}{cc}
\frac{s_{l}}{s_{l-k}} \frac{(-l+k) !}{(-l) !} U \frac{\partial^{-k}}{\partial z^{-k}} U g & \text { if } l \leq k \\
(-1)^{k} \frac{s_{l}}{s_{l-k}} \frac{(l-1) !}{(l-k-1) !} U \frac{\partial^{-k}}{\partial z^{-k}} U g & \text { if } l>0
\end{array}\right.
$$

This completes the proof.

Proposition 3.2. Suppose that $k, l \in \mathbb{Z}$ and let $g$ be the monomial of the form $g(z):=z^{l}$. Then

If $k \geq 0$

$$
\left(H_{\bar{z}^{k}} g\right)(\xi)=\left\{\begin{array}{lc}
\bar{\xi}^{k} \xi^{l}-\frac{s_{l}}{s_{l-k}} \xi^{l-k} & \text { if } l \geq k \text { or if } l<0 \\
\bar{\xi}^{k} \xi^{l} & \text { if } 0 \leq l<k
\end{array}\right.
$$

If $k<0$

$$
\left(H_{\bar{z}^{k}} g\right)(\xi)=\left\{\begin{array}{cc}
\bar{\xi}^{k} \xi^{l}-\frac{s_{l}}{s_{l-k}} \xi^{l-k} & \text { if } l \leq k \text { or if } l>0 \\
\bar{\xi}^{k} \xi^{l} & \text { if } k<l \leq 0
\end{array}\right.
$$

Proof. Since $H_{\bar{f}}(\varphi)=\left(I-P_{\mu_{s}}\right)(\bar{f} \varphi)$ for each holomorphic Laurent polynomial $\varphi$, from Proposition 1 it follows that that the Hankel operator $H_{\bar{z}^{k}}$ can be written in the following form:

Case where $k \geq 0$

$$
\left(H_{\bar{z}^{k}} g\right)(\xi)=\left\{\begin{array}{cc}
\bar{\xi}^{k} g(\xi)-\frac{s_{l}}{s_{l-k}} \frac{(l-k) !}{l !} \frac{\partial^{k}}{\partial z^{k}} g(\xi) & \text { if } l \geq k \\
\bar{\xi}^{k} g(\xi)-(-1)^{k} \frac{(-l-1) !}{(-l+k-1) !} \frac{s_{l}}{s_{l-k}} \frac{\partial^{k}}{\partial z^{k}} g & \text { if } l<0 .
\end{array}\right.
$$

In particular, for $g=z^{l}, l \in \mathbb{Z}$ the latter equality reduces to

$$
\left(H_{\bar{z}^{k}} g\right)(\xi)=\left\{\begin{array}{cc}
\bar{\xi}^{k} \xi^{l}-\frac{s_{l}}{s_{l-k}} \xi^{l-k} & \text { if } l \geq k \text { or if } l<0 \\
\bar{\xi}^{k} \xi^{l} & \text { if } 0 \leq l<k
\end{array}\right.
$$

Case where $k<0$ :

In this case the Hankel operator $H_{\bar{z}^{k}}$ can be expressed in the following form:

$$
\left(H_{\bar{z}^{k}} g\right)(\xi)=\left\{\begin{array}{cl}
\bar{\xi}^{k} g(\xi)-\frac{s_{l}}{s_{l-k}} \frac{(-l+k) !}{(-l) !} U \frac{\partial^{-k}}{\partial z^{-k}} U g & \text { if } l \leq k \\
\bar{\xi}^{k} g(\xi)-(-1)^{k} \frac{s_{l}}{s_{l-k}} \frac{(l-1) !}{(l-k-1) !} U \frac{\partial^{-k}}{\partial z^{-k}} U g & \text { if } l>0
\end{array}\right.
$$

especially, for $g=z^{l}, l \in \mathbb{Z}$, we have

$$
\left(H_{\bar{z}^{k}} g\right)(\xi)=\left\{\begin{array}{lc}
\bar{\xi}^{k} \xi^{l}-\frac{s_{l}}{s_{l-k}} \xi^{l-k} & \text { if } l \leq k \text { or if } l>0 \\
\bar{\xi}^{k} \xi^{l} & \text { if } k<l \leq 0 .
\end{array}\right.
$$


The proof is now complete.

Lemma 3.3. Suppose that $u, v$ and $g$ are holomorphic Laurent polynomials. Then

$$
H_{\bar{v}}^{*} H_{\bar{u}} g=P_{\mu_{s}}(v \bar{u} g)-v P_{\mu_{s}}(\bar{u} g)
$$

Proof. In fact, for $h \in \mathcal{A}^{2}(s)$

$$
\begin{aligned}
\left\langle H_{\bar{u}} g, H_{\bar{v}} h\right\rangle_{L_{\left(\mu_{s}\right)}^{2}} & =\left\langle\bar{u} g-P_{\mu_{s}}(\bar{u} g), \bar{v} h-P_{\mu_{s}}(\bar{v} h)\right\rangle_{L_{\left(\mu_{s}\right)}^{2}} \\
& =\langle v \bar{u} g, h\rangle_{L_{\left(\mu_{s}\right)}^{2}}-\left\langle P_{\mu_{s}}(\bar{u} g), \bar{v} h\right\rangle_{L_{\left(\mu_{s}\right)}^{2}}+\left\langle\left(P_{\mu_{s}}-I\right) \bar{u} g, P_{\mu_{s}}(\bar{v} h)\right\rangle_{L_{\left(\mu_{s}\right)}^{2}} .
\end{aligned}
$$

Since $P_{\mu_{s}}(\bar{v} h) \in \mathcal{A}^{2}\left(\mu_{s}\right)$ and $\left(P_{\mu_{s}}-I\right)(\bar{u} g)$ is orthogonal to $\mathcal{A}^{2}\left(\mu_{s}\right)$ we see that

$$
H_{\bar{v}}^{*} H_{\bar{u}} g=P_{\mu_{s}}(v \bar{u} g)-v P_{\mu_{s}}(\bar{u} g) .
$$

and thus the proof of the lemma follows.

Next, we shall establish the expression of the operator $H_{\bar{z}^{k}}^{*} H_{\bar{z}^{k}}$.

Proposition 3.4. Suppose that $k \in \mathbb{Z}$ and let $g$ be holomorphic Laurent polynomial. Then

$$
H_{\bar{z}^{k}}^{*} H_{\bar{z}^{k}}(g)=\left\{\begin{array}{c}
\sum_{l \geq 0} \hat{g}(l)\left[\frac{s_{l+k}}{s_{l}} \frac{(l) !}{(l+k) !} \frac{\partial^{k}}{\partial z^{k}}\left(z^{k+l}\right)-\frac{s_{l}}{s_{l-k}} \frac{(l-k) !}{l !} z^{k} \frac{\partial^{k}}{\partial z^{k}} z^{l}\right] \\
+(-1)^{k} \sum_{l<-k} \hat{g}(l)\left[\frac{(-l-k-1) !}{(-l-1) !} \frac{s_{l+k}}{s_{l}} \frac{\partial^{k}}{\partial z^{k}}\left(z^{k+l} g\right)-\frac{(-l-1) !}{(-l+k-1) !} \frac{s_{l}}{s_{l-k}} z^{k} \frac{\partial^{k}}{\partial z^{k}} z^{l}\right]
\end{array}\right.
$$

if $k \geq 0$ and,

$H_{\bar{z}^{k}}^{*} H_{\bar{z}^{k}}(g)=\left\{\begin{array}{c}\sum_{l \leq 0} \hat{g}(l)\left[\frac{s_{l+k}}{s_{l}} \frac{(-l) !}{(-l-k) !} U \frac{\partial^{-k}}{\partial z^{-k}} U\left(z^{k+l}\right)-\frac{s_{l}}{s_{l-k}} \frac{(-l+k) !}{(-l) !} z^{k} U \frac{\partial^{-k}}{\partial z^{-k}} U z^{l}\right] \\ +(-1)^{k} \sum_{l>-k} \hat{g}(l)\left[\frac{s_{l+k}}{s_{l}} \frac{(l+k-1) !}{(l-1) !} U \frac{\partial^{-k}}{\partial z^{-k}} U\left(z^{k+l}\right)-\frac{s_{l}}{s_{l-k}} \frac{(l-1) !}{(l-k-1) !} U \frac{\partial^{-k}}{\partial z^{-k}} U z^{k}\right]\end{array}\right.$

otherwise.

Proof. We first notice that if $k \geq 0$

$$
P_{\mu_{s}}\left(z^{p} \bar{z}^{k} g\right)= \begin{cases}\frac{s_{l+p}}{s_{l+p-k}} \frac{(l+p-k) !}{(l+p) !} \frac{\partial^{k}}{\partial z^{k}}\left(z^{p} g\right) & \text { if } l+p \geq k \\ (-1)^{k} \frac{(-l-p-1) !}{(-l-p+k-1) !} \frac{s_{l+p}}{s_{l+p-k}} \frac{\partial^{k}}{\partial z^{k}}\left(z^{p} g\right) & \text { if } l+p<0\end{cases}
$$

and for $k<0$, we have

$$
P_{\mu_{s}}\left(z^{p} \bar{z}^{k} g\right)= \begin{cases}\frac{s_{l+p}}{s_{l+p-k}} \frac{(-l-p+k) !}{(-l-p) !} U \frac{\partial^{-k}}{\partial z^{-k}} U\left(z^{p} g\right) & \text { if } l+p \leq k \\ (-1)^{k} \frac{s_{l+p}}{s_{l+p-k}} \frac{(l+p-1) !}{(l+p-k-1) !} U \frac{\partial^{-k}}{\partial z^{-k}} U\left(z^{p} g\right) & \text { if } l+p>0 .\end{cases}
$$

Then we use the previous lemma to conclude. 
In particular, for $g=\xi^{l}, l \in \mathbb{Z}$ we deduce that :

For $k \geq 0$,

$$
\left(H_{\bar{z}^{k}}^{*} H_{\bar{z}^{k}} g\right)(\xi)=\left\{\begin{array}{lc}
\left(\frac{s_{l+k}}{s_{l}}-\frac{s_{l}}{s_{l-k}}\right) \xi^{l} & \text { if } l \geq k \\
\frac{s_{l+k}}{s_{l}} \xi^{l} & \text { if } 0 \leq l<k \\
-\frac{s_{l}}{s_{l-k}} \xi^{l} & \text { if }-k \leq l<0 \\
\left(\frac{s_{l+k}}{s_{l}}-\frac{s_{l}}{s_{l-k}}\right) \xi^{l} & \text { if } l<-k,
\end{array}\right.
$$

and for $k<0$,

$$
\left(H_{\bar{z}^{k}}^{*} H_{\bar{z}^{k}} g\right)(\xi)=\left\{\begin{array}{lc}
\left(\frac{s_{l+k}}{s_{l}}-\frac{s_{l}}{s_{l-k}}\right) \xi^{l} & \text { if } l \leq k \\
\frac{s_{l+k}}{s_{l}} \xi^{l} & \text { if } k<l \leq 0 \\
-\frac{s_{l}}{s_{l-k}} \xi^{l} & \text { if } 0<l \leq-k \\
\left(\frac{s_{l+k}}{s_{l}}-\frac{s_{l}}{s_{l-k}}\right) \xi^{l} & \text { if } l>-k
\end{array}\right.
$$

This result allows us to obtain the eigenvalues associated to the operator $H_{\bar{z}^{k}}^{*} H_{\bar{z}^{k}}$ and to study its spectral properties. This leads to the following result.

Theorem 3.5. Suppose that $f$ is holomorphic Laurent polynomial. Then

1) The operator $H_{\bar{f}}$ is bounded if and only if

$$
\sup _{n \in \mathbb{N}}\left(\frac{s_{n+k}}{s_{n}}-\frac{s_{n}}{s_{n-k}}\right)<+\infty \text { and } \sup _{n \in \mathbb{N}}\left(\frac{\widetilde{s}_{n+k}}{\widetilde{s}_{n}}-\frac{\widetilde{s}_{n}}{\widetilde{s}_{n-k}}\right)<+\infty, \forall k \in \operatorname{spec}(f) .
$$

2) The operator $H_{\bar{f}}$ is compact if and only if

$$
\lim _{n \rightarrow+\infty}\left(\frac{s_{n+k}}{s_{n}}-\frac{s_{n}}{s_{n-k}}\right)=0 \text { and } \lim _{n \rightarrow+\infty}\left(\frac{\widetilde{s}_{n+k}}{\widetilde{s}_{n}}-\frac{\widetilde{s}_{n}}{\widetilde{s}_{n-k}}\right)=0, \forall k \in \operatorname{spec}(f) .
$$

Proof. It suffices to observe that the sequence $\left(\lambda_{l}\right)$ of eigenvalues of the operator $H_{\bar{z}^{k}}^{*} H_{\bar{z}^{k}}$ can be written as follows :

If $k \geq 0$

$$
\lambda_{l}=\left\{\begin{array}{lc}
\left(\frac{s_{l+k}}{s_{l}}-\frac{s_{l}}{s_{l-k}}\right) & \text { if } l \geq k \\
\frac{s_{l+k}}{s_{l}} & \text { if } 0 \leq l<k \\
-\frac{\tilde{s}_{-l}}{\tilde{s}_{-(l-k)}} & \text { if }-k \leq l<0 \\
\left(\frac{\tilde{s}_{-(l+k)}}{\tilde{s}_{-l}}-\frac{\widetilde{s}_{-l}}{\tilde{s}_{-(l-k)}}\right) & \text { if } l<-k
\end{array}\right.
$$


If $k<0$,

$$
\lambda_{l}= \begin{cases}\left(\frac{\widetilde{s}_{-(l+k)}}{\widetilde{s}_{-l}}-\frac{\widetilde{s}_{-l}}{\widetilde{s}_{-(l-k)}}\right) & \text { if } l \leq k \\ \frac{\widetilde{s}_{-(l+k)}}{\widetilde{s}_{-l}} & \text { if } k<l \leq 0 \\ -\frac{s_{l}}{s_{l-k}} & \text { if } 0<l \leq-k \\ \left(\frac{s_{l+k}}{s_{l}}-\frac{s_{l}}{s_{l-k}}\right) & \text { if } l>-k\end{cases}
$$

The proof of the theorem follows since the operator $H_{\bar{z}^{k}}^{*} H_{\bar{z}^{k}}$ is bounded if and only if the sequence sup $\left|\lambda_{l}\right|$ is bounded and $H_{\bar{z}^{k}}^{*} H_{\bar{z}^{k}}$ is compact if and only if the sequence $\sup \left|\lambda_{l}\right|$ tends to zero.

We recall that if $E$ and $F$ are two Hilbert spaces and $0<p<\infty$, then the $p^{\text {th }}$-Schatten class $\mathcal{S}_{p}(E, F)$, is the space of all compact operators $T$ from $E$ to $F$ such that the the sequence of singular values of $T,\left\{s_{n}(T)\right\}_{n \in \mathbb{N}}$, satisfies

$$
\|T\|_{\mathcal{S}_{p}(E, F)}:=\left(\sum_{n \in \mathbb{N}}\left[s_{n}(T)\right]^{p}\right)^{\frac{1}{p}}<+\infty
$$

with $s_{n}(T)=\left[\lambda_{n}\left(T^{*} T\right)\right]^{\frac{1}{2}}$, for all $n \in \mathbb{N}$.

The class $\mathcal{S}_{1}$ is the trace class. Specifically, if $T \in \mathcal{S}_{1}$, then the trace of $T$, is noted

$$
\operatorname{tr}(T):=\|T\|_{\mathcal{S}_{1}}=\sum_{n \in \mathbb{N}} s_{n}(T)
$$

The following two theorems are well-known facts and reduce the study of the membership of an operator $T$ to the Schatten class $\mathcal{S}_{p}$, to membership of $\left(T^{*} T\right)^{\frac{p}{2}}$ to the class $\mathcal{S}_{1}$.

Theorem 3.6. Let $E$ be a Hilbert space and $p>0$. If $T$ is a positif compact operator from $E$ to $E$, then $T \in \mathcal{S}_{p}(E, E)$ if and only if $T^{p} \in \mathcal{S}_{1}(E, E)$. In addition, we have

$$
\|T\|_{\mathcal{S}_{p}(E, E)}^{p}=\left\|T^{p}\right\|_{\mathcal{S}_{1}(E, E)}
$$

Theorem 3.7. Let $E$ and $F$ be two Hilbert spaces and $1 \leq p<\infty$. If $T$ is a compact operator from $E$ to $F$, then $T \in \mathcal{S}_{p}(E, F)$ if and only if $\left(T^{*} T\right)^{\frac{p}{2}} \in$ $\mathcal{S}_{1}(E, E)$. In addition, we have

$$
\|T\|_{\mathcal{S}_{p}(E, F)}^{p}=\left\|\left(T^{*} T\right)^{\frac{p}{2}}\right\|_{\mathcal{S}_{1}(E, E)}
$$

See Meise and Vogt [25] or Zhu [36] for the above material.

The following theorem characterizes the membership of the operator $H_{\bar{f}}$ to the Schatten class $\mathcal{S}_{p}\left(\mathcal{A}^{2}\left(\mu_{s}\right), L^{2}\left(\mu_{s}\right)\right)$.

Theorem 3.8. Suppose that $f$ is holomorphic Laurent polynomial. Then the operator $H_{\bar{f}}$ is in Schatten class $\mathcal{S}_{p}\left(\mathcal{A}^{2}\left(\mu_{s}\right), L^{2}\left(\mu_{s}\right)\right)$ if and only if

$$
\sum_{n \in \mathbb{N}}\left(\frac{s_{n+k}}{s_{n}}-\frac{s_{n}}{s_{n-k}}\right)^{\frac{p}{2}}<+\infty \text { and } \sum_{n \in \mathbb{N}}\left(\frac{\widetilde{s}_{n+k}}{\widetilde{s}_{n}}-\frac{\widetilde{s}_{n}}{\widetilde{s}_{n-k}}\right)^{\frac{p}{2}}<+\infty, \forall k \in \operatorname{spec}(f) .
$$




\section{APPLICATION}

In this section we consider the space $\mathcal{A}^{2}\left(\mu_{m}\right)$ defined in the introduction. We remind that $\mu_{m}=e^{-|z|^{m}-\frac{1}{|z|^{\mid m}}} d \delta(z), m>0$ and $d A(z)$ is the Lebesgue measure in complex plane.

We set $T=H_{\bar{z}^{k}}^{*} H_{\bar{z}^{k}}$, then $T$ is well-defined on the space of holomorphic Laurent polynomials which is dense in $\mathcal{A}^{2}\left(\mu_{m}\right)$. In addition, the eigenvalues corresponding to $T$ are given by relations (3.1) and (3.2).

The moments of the measure $\mu_{m}$ are given by

$$
s_{d}=\int_{0}^{+\infty} t^{d} \exp \left(-t^{m}-\frac{1}{t^{m}}\right) d t, d \in \mathbb{Z} .
$$

In addition, they satisfy $\widetilde{s}_{n}=s_{-n}=s_{n-2}$ for all $n \in \mathbb{N}$ and we have

$$
s_{n}=\int_{0}^{+\infty} t^{n} \exp \left(-t^{m}-\frac{1}{t^{m}}\right) d t=\frac{1}{m} \int_{0}^{+\infty} t^{\frac{n+1}{m}-1} \exp \left(-t-\frac{1}{t}\right) d t .
$$

Therefore $s_{n}=\frac{2}{m} K_{\frac{n+1}{m}}(2)$ with $K_{\nu}$ is the function defined by

$$
K_{\nu}(z)=\int_{0}^{+\infty} \exp (z \cosh (t)) \cosh (\nu t) d t
$$

This function is called modified Bessel function of the second kind or Macdonald function. It is a solution of a modified Bessel differential equation and has been studied by several authors see [2, 14, 24, 30, 31, 32] and [33]. More precisely, from relation 1.66 in [4] we have

$$
K_{\nu}(z)=\frac{1}{2}\left(\frac{z}{2}\right)^{\nu} \int_{0}^{+\infty} t^{-\nu-1} \exp \left(-t-\frac{z^{2}}{4 t}\right) d t .
$$

The eigenvalue associated to the operator $T$ corresponding to the eigenvector $\xi^{l}$ is given by the relations (3.1) and (3.2). This allows us to study asymptotically these eigenvalues and to deduce the spectral properties of the operator $T$. To achieve this, we need the following lemma which provides the approximation of Macdonald function as $\nu \rightarrow \infty$.

Lemma 4.1. Let $\alpha, \beta$ and $\nu$ be real numbers, for $\nu \rightarrow+\infty$ and $z$ fixed we have:

$$
\frac{K_{\nu+\alpha}(z)}{K_{\nu+\beta}(z)}=\left(\frac{1}{2} z\right)^{\beta-\alpha} \nu^{\alpha-\beta}\left(1+\frac{(\alpha-\beta)(\alpha+\beta-1)}{2 \nu}+\mathcal{O}\left(\frac{1}{\nu^{2}}\right)\right)+o(1)
$$

Proof. Let $\alpha, \beta$ be real numbers and $\nu \rightarrow+\infty$, . By [30, Relation 9.37 page 234], we have the following asymptotic relation

$$
K_{\nu} \sim \frac{1}{2} \Gamma(\nu)\left(\frac{1}{2}\right)^{-\nu} \operatorname{Re}(\nu)>0 \text { and } z \rightarrow 0
$$

This estimation remains true if $z$ is fixed and $\nu \rightarrow+\infty$.

We also use another estimate of $K_{\nu}(z)$ when $z$ is fixed and $\nu \rightarrow+\infty$, see [11, page 44], when $\nu \rightarrow+\infty$ and $z$ is positive we have

$$
K_{\nu}(z) \sim \sqrt{\frac{\pi}{2}} \frac{2^{\nu} \nu^{\nu-\frac{1}{2}} e^{-\nu}}{z^{\nu}}
$$


Using the fact that

$$
\frac{\Gamma(\nu+\alpha)}{\Gamma(\nu+\alpha)}=\nu^{\alpha-\beta}\left(1+\frac{(\alpha-\beta)(\alpha+\beta-1)}{2 \nu}+\mathcal{O}\left(\frac{1}{\nu^{2}}\right)\right), \text { when } \nu \rightarrow+\infty,
$$

then we have

$$
\frac{K_{\nu+\alpha}(z)}{K_{\nu+\beta}(z)}=\left(\frac{1}{2} z\right)^{\beta-\alpha} \nu^{\alpha-\beta}\left(1+\frac{(\alpha-\beta)(\alpha+\beta-1)}{2 \nu}+\mathcal{O}\left(\frac{1}{\nu^{2}}\right)\right)+o(1)
$$

and thus the proof of the lemma is complete.

It is well-known that $H_{\bar{z}^{k}}$ is bounded (resp. compact) if and only if $H_{\bar{z}^{k}}^{*} H_{\bar{z}^{k}}$ is bounded (resp.compact). Now we state the following spectral properties of the operator $H_{\bar{z}^{k}}$ as :

Theorem 4.2. Suppose that $k \in \mathbb{Z}$ and $m$ is a positive real number. Then

1) The Hankel operator $H_{\bar{z}^{k}}$ is bounded on the space $\mathcal{A}^{2}\left(\mu_{m}\right)$ if and only if $k \leq m$.

2) The Hankel operator $H_{\bar{z}^{k}}$ is compact on the space $\mathcal{A}^{2}\left(\mu_{m}\right)$ if and only if $k<m$.

3) The Hankel operator $H_{\bar{z}^{k}}$ is in Schatten class $\mathcal{S}_{p}\left(\mathcal{A}^{2}\left(\mu_{m}\right), L^{2}\left(\mu_{m}\right)\right), p>0$, if and only if $p k<m(p-2)$.

Proof. Since the moments are symmetric, the operator $H_{\bar{z}^{k}}$ is bounded if and only if

$$
\sup _{n \in \mathbb{N}}\left(\frac{s_{n+k}}{s_{n}}-\frac{s_{n}}{s_{n-k}}\right)<+\infty .
$$

On the other hand, a little computing shows that

$$
\begin{aligned}
\left(\frac{s_{n+k}}{s_{n}}-\frac{s_{n}}{s_{n-k}}\right) & =\frac{K_{\frac{n+k+1}{m}}(2)}{K_{\frac{n+1}{m}}(2)}-\frac{K_{\frac{n+1}{m}}(2)}{K_{\frac{n-k+1}{m}}(2)} \\
& =\frac{K_{\frac{n+1}{m}+\frac{k}{m}}(2)}{K_{\frac{n+1}{m}}(2)}-\frac{K_{\frac{n+1}{m}}(2)}{K_{\frac{n+1}{m}-\frac{k}{m}}(2)} .
\end{aligned}
$$

Therefore, Lemma 4.1 implies that

$$
\frac{K_{\frac{n+1}{m}+\frac{k}{m}}(2)}{K_{\frac{n+1}{m}}(2)}=\left(\frac{1}{m}\right)^{\frac{k}{m}}(n+1)^{\frac{k}{m}}\left(1+\frac{k(k-m)}{2 m(n+1)}+\mathcal{O}\left(\frac{1}{(n+1)^{2}}\right)\right)
$$

and

$$
\frac{K_{\frac{n+1}{m}}(2)}{K_{\frac{n+1}{m}-\frac{k}{m}}(2)}=\left(\frac{1}{m}\right)^{\frac{k}{m}}(n+1)^{\frac{k}{m}}\left(1-\frac{k(k+m)}{2 m(n+1)}+\mathcal{O}\left(\frac{1}{(n+1)^{2}}\right)\right) .
$$

This shows that

$$
\begin{aligned}
\left(\frac{s_{n+k}}{s_{n}}-\frac{s_{n}}{s_{n-k}}\right)= & \left(\frac{1}{m}\right)^{\frac{k}{m}}(n+1)^{\frac{k}{m}}\left(\left(1+\frac{k(k-m)}{2 m(n+1)}+\mathcal{O}\left(\frac{1}{(n+1)^{2}}\right)\right)\right. \\
& \left.-\left(1-\frac{k(k+m)}{2 m(n+1)}+\mathcal{O}\left(\frac{1}{(n+1)^{2}}\right)\right)\right) \\
= & \left(\frac{1}{m}\right)^{\frac{k}{m}}(n+1)^{\frac{k}{m}}\left(\frac{k^{2}}{m(n+1)}+\mathcal{O}\left(\frac{1}{(n+1)^{2}}\right)\right) .
\end{aligned}
$$

Thus the Hankel operator $H_{\bar{z}^{k}}$ is bounded if and only if $\frac{k}{m}-1 \leq 0$, and it is compact if and only if $\frac{k}{m}-1<0$. 
We observe that $\lambda_{n} \simeq(n+1)^{\frac{k}{m}-1}$. Thus the Hankel operator $H_{\bar{z}^{k}}$ is in the Schatten class $\mathcal{S}_{p}\left(\mathcal{A}^{2}\left(\mu_{m}\right), L^{2}\left(\mu_{m}\right)\right), p>0$, if and only if $p k<m(p-2)$.

Next, denote by $\mathcal{M}$ the subspace of all functions $f \in \mathcal{A}^{2}\left(\mu_{m}\right)$ such that $H_{\bar{f}}$ is bounded on $\mathcal{A}^{2}\left(\mu_{m}\right)$. We equip this space with semi-norm

$$
\|f\|:=\left\|H_{\bar{f}}\right\|+|f(0)|
$$

The subspace of $\mathcal{M}$ consisting of those functions $f$ such that $H_{\bar{f}}$ is a compact operator will be denoted by $\mathcal{M}_{\infty}$. Is not hard to see that $\mathcal{M}_{\infty}$ is a closed subspace of $\mathcal{M}$.

If $p>0$, we denote by $\mathcal{M}_{p}$ the subspace of all functions $f \in \mathcal{M}$ such that $H_{\bar{f}}$ is in the Schatten $\mathcal{S}_{p}\left(\mathcal{A}^{2}\left(\mu_{m}\right), L^{2}\left(\mu_{m}\right)\right)$. We equip this space with semi-norm

$$
\|f\|:=\left\|H_{\bar{f}}\right\|_{\mathcal{S}_{p}}+|f(0)|
$$

For $\theta \in \mathbb{R}$, we let $R_{\theta}$ be the rotation on $\mathbb{C}$ given by $R_{\theta}(z)=e^{i \theta} z$ for all $z \in \mathbb{C}$. This induces a linear transformation defined on functions $f$ defined on a $R_{\theta}$-invariant subset of $\mathbb{C}$ of by

$$
R_{\theta} f:=f \circ R_{\theta}
$$

Lemma 4.3. Let $\theta \in \mathbb{R}$. Then the operator $R_{\theta}$ is a unitary isometry from $L^{2}\left(\mu_{m}\right)$ onto itself, and from $\mathcal{A}^{2}(\mu)$ onto itself. Moreover we have the following results

1) If $f \in \mathcal{M}, R_{\theta} f \in \mathcal{M}$ and $\left\|R_{\theta} f\right\|_{\mathcal{M}}=\|f\|_{\mathcal{M}}$.

2) If $f \in \mathcal{M}_{\infty}, R_{\theta} f \in \mathcal{M}_{\infty}$.

3) If $f \in \mathcal{M}_{p}, R_{\theta} f \in \mathcal{M}_{p}$ and $\left\|R_{\theta} f\right\|_{\mathcal{M}_{p}}=\|f\|_{\mathcal{M}_{p}}$.

Proof. It is clear that the operator $R_{\theta} f$ is a unitary isometry from $L^{2}\left(\mu_{m}\right)$ onto itself, and from $\mathcal{A}^{2}\left(\mu_{m}\right)$ onto itself. Let $f \in \mathcal{M}$ and $\theta \in \mathbb{R}$. Then $R_{\theta} f \in \mathcal{A}^{2}\left(\mu_{m}\right)$. Moreover, if $g$ is holomorphic Laurent polynomial, then

$$
\begin{aligned}
H_{\overline{R_{\theta} f}}(g)(z) & =\left(I-P_{\mu_{m}}\right)\left(\overline{R_{\theta} f} g\right)(z)=\left(I-P_{\mu_{m}}\right)\left(\bar{f}\left(R_{\theta} z\right) g(z)\right) \\
& =\left(I-P_{\mu_{m}}\right)\left(\bar{f}\left(R_{\theta} z\right) g\left(R_{-\theta} R_{\theta} z\right)\right) \\
& =\left(I-P_{\mu_{m}}\right)\left(\bar{f} R_{-\theta} g\right)\left(R_{\theta} z\right) \\
& =\left(R_{\theta} H_{\bar{f}} R_{-\theta}\right)(g)(z)
\end{aligned}
$$

showing that

$$
\left\|H_{\overline{R_{\theta} f}}\right\|=\left\|H_{\bar{f}}\right\|
$$

and thus

$$
\left\|R_{\theta} f\right\|_{\mathcal{M}}=\|f\|_{\mathcal{M}}
$$

The proof of parts 2) and 3) of the lemma is similar.

Lemma 4.4. The spaces $\mathcal{M}$ and $\mathcal{M}_{p}, p \geq 1$, are Banach spaces. If $0<p<1$ then $\mathcal{M}_{p}$ is quasi-Banach space. 
Proof. Let $\left(f_{q}\right)_{q \in \mathbb{N}}$ be a Cauchy sequence in $\mathcal{M}$. Without loss of generality we may assume that $f_{q}(0)=0$ for all $q \in \mathbb{N}$. Then the sequence $\left(H_{\bar{f}_{q}}\right)_{q \in \mathbb{N}}$ is a Cauchy sequence of bounded operators on $\mathcal{A}^{2}\left(\mu_{m}\right)$. Therefore, there is an operator $T \in$ $\mathcal{A}^{2}\left(\mu_{m}\right)$ such that $\left(H_{\bar{f}_{q}}\right)_{q \in \mathbb{N}}$ converges to $T$ in the norm operator. Let $f:=\overline{T(1)}$ be the conjugate of the image $T(1)$ of the constant function 1 . Since $H_{\bar{f}_{q}}(1)=\bar{f}_{q}$, it follows that

$$
\begin{aligned}
\left\|f_{q}-f\right\|_{L^{2}\left(\mu_{m}\right)} & =\left\|\overline{f_{q}}-T(1)\right\|_{L^{2}\left(\mu_{m}\right)}=\left\|H_{\bar{f}_{q}}(1)-T(1)\right\|_{L^{2}\left(\mu_{m}\right)} \\
& \leq\left\|H_{\bar{f}_{q}}-T\right\|_{L^{2}\left(\mu_{m}\right)} \cdot\|1\|_{L^{2}\left(\mu_{m}\right)},
\end{aligned}
$$

which shows that $\lim _{q \rightarrow+\infty}\left\|f_{q}-f\right\|_{L^{2}\left(\mu_{m}\right)}=0$ and thus $f \in \mathcal{A}^{2}\left(\mu_{m}\right)$.

It remains to show that the operator $H_{\bar{f}}$ is bounded. To do so, let $g$ be a holomorphic Laurent polynomial. Then

$$
\left|P_{\mu_{m}}\left(\left(\bar{f}-\bar{f}_{q}\right) g\right)(z)\right| \leq\left\|f_{q}-f\right\|_{L^{2}\left(\mu_{m}\right)}\left\|g K_{m}(z, .)\right\|_{L^{2}\left(\mu_{m}\right)} .
$$

Therefore $\lim _{q \rightarrow+\infty} P_{\mu_{m}}\left(\left(\bar{f}-\bar{f}_{q}\right) g\right)(z)=0$. Since $\lim _{q \rightarrow+\infty}\left\|f_{q}-f\right\|_{L^{2}\left(\mu_{m}\right)}=0$, it follows that

$$
\lim _{q \rightarrow+\infty}\left(H_{\bar{f}_{q}}-H_{\bar{f}}\right)(g)(z)=0
$$

showing that $T=H_{\bar{f}}$ and hence $\mathcal{M}$ is a Banach space.

The proof of the rest of lemma is similar.

Lemma 4.5. Suppose that $f \in \mathcal{A}^{2}\left(\mu_{m}\right)$ and $H_{\bar{f}}$ is bounded on $\mathcal{A}^{2}\left(\mu_{m}\right)$. Then for all $g \in \mathcal{A}^{2}\left(\mu_{m}\right)$, the mapping $\theta \mapsto H_{\overline{R_{\theta} f}}(g)$ is continuous from $[0,2 \pi]$ into $L^{2}\left(\mu_{m}\right)$.

Proof. Let $g \in \mathcal{A}^{2}\left(\mu_{m}\right)$ written as $g(z)=\sum_{k \in \mathbb{Z}} a_{k} z^{k}$. If $\theta, \varphi \in[0,2 \pi]$, Then a little computing shows that

$$
\begin{aligned}
H_{\overline{R_{\theta} f}}-H_{\overline{R_{\varphi} f}}= & R_{\theta} H_{\bar{f}} R_{-\theta}-R_{\varphi} H_{\bar{f}} R_{-\varphi} \\
& =R_{\theta} H_{\bar{f}} R_{-\theta}-R_{\theta} H_{\bar{f}} R_{-\varphi}+R_{\theta} H_{\bar{f}} R_{-\varphi}-R_{\varphi} H_{\bar{f}} R_{-\varphi} \\
& =R_{\theta} H_{\bar{f}}\left(R_{-\theta}-R_{-\varphi}\right)+\left(R_{\theta}-R_{\varphi}\right) H_{\bar{f}} R_{-\varphi} .
\end{aligned}
$$

Thus

$$
\left\|H_{\overline{R_{\theta} f}}(g)-H_{\overline{R_{\varphi} f}}(g)\right\|_{L^{2}\left(\mu_{m}\right)} \leq\left\|H_{\bar{f}}\right\|\left\|\left(R_{-\theta}-R_{-\varphi}\right) g\right\|_{L^{2}\left(\mu_{m}\right)}+\left\|\left(R_{\theta}-R_{\varphi}\right) H_{\bar{f}} R_{-\varphi} g\right\|_{L^{2}\left(\mu_{m}\right)} .
$$

On the other hand,

$$
\begin{aligned}
\left\|\left(R_{-\theta}-R_{-\varphi}\right) g\right\|_{L^{2}\left(\mu_{m}\right)}^{2} & =\left\|g\left(R_{-\theta} z\right)-g\left(R_{-\varphi} z\right)\right\|_{L^{2}\left(\mu_{m}\right)}^{2} \\
& =\sum_{k \in \mathbb{Z}} a_{k}^{2}\left\|\left(R_{-\theta} z\right)^{k}-\left(R_{-\varphi} z\right)^{k}\right\|_{L^{2}\left(\mu_{m}\right)}^{2} \\
& =\sum_{k \in \mathbb{Z}} a_{k}^{2} s_{k}\left|e^{-i k \theta}-e^{-i k \varphi}\right|^{2}<+\infty .
\end{aligned}
$$

Therefore

$$
\lim _{\theta \rightarrow \varphi}\left\|\left(R_{-\theta}-R_{-\varphi}\right) g\right\|_{L^{2}\left(\mu_{m}\right)}=0
$$


A similar argument shows that

$$
\lim _{\theta \rightarrow \varphi}\left\|\left(R_{\theta}-R_{\varphi}\right) H_{\bar{f}} R_{-\varphi} g\right\|_{L^{2}\left(\mu_{m}\right)}=0 .
$$

Therefore,

$$
\lim _{\theta \rightarrow \varphi}\left\|H_{\overline{R_{\theta} f}}(g)-H_{\overline{R_{\varphi} f}}(g)\right\|_{L^{2}\left(\mu_{m}\right)}=0,
$$

which completes the proof

Lemma 4.6. Let $f \in \mathcal{A}^{2}\left(\mu_{m}\right)$.

1) If $f \in \mathcal{M}$, then for any $k \in \operatorname{spec}(f)$, the monomial $z^{k}$ is in $\mathcal{M}$.

2) If $f \in \mathcal{M}_{\infty}$, then for any $k \in \operatorname{spec}(f)$, the monomial $z^{k}$ is in $\mathcal{M}_{\infty}$.

3) If $p \geq 1$ and $f \in \mathcal{M}_{p}$, then for any $k \in \operatorname{spec}(f)$, the monomial $z^{k}$ is in $\mathcal{M}_{p}$.

Proof. Suppose that $f \in \mathcal{M}$ and write $f(z)=\sum_{k \in \mathbb{Z}} a_{k} z^{k}$. Then is clear that

$$
a_{k} z^{k}=\frac{1}{2 \pi} \int_{0}^{2 \pi} f\left(R_{\theta} z\right) e^{-i k \theta} d \theta, \forall z \in \mathbb{C}^{*} .
$$

Let $g$ be a holomorphic Laurent polynomial, and $h \in L^{2}\left(\mu_{m}\right)$. By Fubini's theorem we see that

$$
\frac{1}{2 \pi} \int_{0}^{2 \pi}\left\langle H_{\overline{R_{\theta} f}}(g), h\right\rangle_{L^{2}\left(\mu_{m}\right)} e^{-i k \theta} d \theta=\left\langle H_{\overline{a_{k} z^{k}}}(g), h\right\rangle_{L^{2}\left(\mu_{m}\right)} .
$$

By Lemmas 4.3 and 4.4, there exists a constant $C$ such that

$$
\left\|H_{\overline{a_{k} z^{k}}}(g)\right\|_{L^{2}\left(\mu_{m}\right)} \leq C\|g\|_{L^{2}\left(\mu_{m}\right)}
$$

Thus $H_{\overline{a_{k} z^{k}}}$ is bounded and $a_{k} z^{k} \in \mathcal{M}$. Therefore, $z^{k} \in \mathcal{M}$ for all $k \in \operatorname{spec}(f)$.

Suppose now that $f \in \mathcal{M}_{\infty}$ and let $\left(g_{q}\right)_{q \in \mathbb{N}}$ be a sequence in $\mathcal{A}^{2}\left(\mu_{m}\right)$ wich converges weakly to 0 . Since $H_{\overline{R_{\theta} f}}$ is a compact operator, we have that

$$
\lim _{q \rightarrow+\infty}\left\|H_{\overline{R_{\theta} f}}\left(g_{q}\right)\right\|_{L^{2}\left(\mu_{m}\right)}=0, \forall \theta \in[0,2 \pi] .
$$

But

$$
\left\|H_{\overline{a_{k} z^{k}}}\left(g_{q}\right)\right\|_{L^{2}\left(\mu_{m}\right)} \leq \int_{0}^{2 \pi}\left\|H_{\overline{R_{\theta} f}}\left(g_{q}\right)\right\|_{L^{2}\left(\mu_{m}\right)} d \theta .
$$

This implies that

$$
\lim _{q \rightarrow+\infty}\left\|H \overline{a_{k} z^{k}}\left(g_{q}\right)\right\|_{L^{2}\left(\mu_{m}\right)}=0
$$

and hence $z^{k} \in \mathcal{M}_{\infty}$ whenever $k \in \operatorname{spec}(f)$.

We recall that if $T$ is a compact operator from $\mathcal{A}^{2}\left(\mu_{m}\right)$ to $L^{2}\left(\mu_{m}\right)$ then its singular numbers $\nu_{q}(T), q \in \mathbb{N}$, are given by

$$
\nu_{q}(T):=\inf _{A \in \mathcal{R}_{q}}\|T-A\|
$$

where $\mathcal{R}_{q}$ is the space of all operators from $\mathcal{A}^{2}\left(\mu_{m}\right)$ to $L^{2}\left(\mu_{m}\right)$ with finite rank at most $q$. 
Let $f \in \mathcal{M}_{p}$, then the sequence $\left(\nu_{q}\left(H_{\bar{f}}\right)\right)_{q}$ is in $l^{p}$. Moreover, there are an orthonormal system $\left(u_{q}\right)_{q}$ in $\mathcal{A}^{2}\left(\mu_{m}\right)$ and an orthonormal system $\left(v_{q}\right)_{q}$ in $L^{2}\left(\mu_{m}\right)$ such that

$$
H_{\bar{f}}=\sum_{q=0}^{+\infty} \nu_{q}\left(H_{\bar{f}}\right)\left\langle., u_{q}\right\rangle v_{q}
$$

where the series converges in the operator norm. See Proposition 16.3 in [25]. If $q$ is a positive integer, consider the operators with finite rank at most $q$ given by

$$
A_{q}:=\sum_{j=0}^{q-1} \nu_{j}\left(H_{\bar{f}}\right)\left\langle., u_{k_{j}}\right\rangle v_{k_{j}}
$$

where

$$
u_{k_{j}}(z)=\frac{1}{2 \pi} \int_{0}^{2 \pi}\left(R_{\theta} u_{j}\right)(z) e^{-i k \theta} d \theta \text { et } v_{k_{j}}(z)=\frac{1}{2 \pi} \int_{0}^{2 \pi}\left(R_{\theta} v_{j}\right)(z) e^{-i k \theta} d \theta
$$

The dominated convergence theorem, combined with relations relation (4.1), leads for $h \in L^{2}\left(\mu_{m}\right)$ and $g \in \mathcal{A}^{2}\left(\mu_{m}\right)$ to

$$
\left\langle\left(H_{\overline{a_{k} z^{k}}}(g)-A_{q}\right), h\right\rangle=\frac{1}{2 \pi} \int_{0}^{2 \pi} \sum_{j=q}^{+\infty} \nu_{j}\left(H_{\bar{f}}\right)\left\langle R_{\bar{\theta}} g, u_{j}\right\rangle\left\langle v_{j}, R_{\bar{\theta}} h\right\rangle e^{-i k \theta} d \theta
$$

Due to the fact that the systems $\left(u_{j}\right)_{j}$ and $\left(v_{j}\right)_{j}$ are orthonormal and the sequence $\left(\nu_{j}\left(H_{\bar{f}}\right)\right)_{j}$ is non-increasing, it follows that

$$
\left|\sum_{j=q}^{+\infty} \nu_{j}\left(H_{\bar{f}}\right)\left\langle R_{\bar{\theta}} g, u_{j}\right\rangle\left\langle v_{j}, R_{\bar{\theta}} h\right\rangle\right| \leq \nu_{q}\left(H_{\bar{f}}\right)\|g\|_{\mathcal{A}^{2}\left(\mu_{m}\right)}\|h\|_{L^{2}\left(\mu_{m}\right)},
$$

for all $h \in L^{2}\left(\mu_{m}\right)$ and $g \in \mathcal{A}^{2}\left(\mu_{m}\right)$. Thus

$$
\left.\| H_{\overline{a_{k} z^{k}}}(g)-A_{q}\right) \| \leq \nu_{q}\left(H_{\bar{f}}\right)
$$

This implies that

$$
\nu_{q}\left(H_{\overline{a_{k} z^{k}}}\right) \leq \nu_{q}\left(H_{\bar{f}}\right) .
$$

Consequently, $z^{k} \in \mathcal{M}_{p}$ for all $k \in \operatorname{spec}(f)$.

Now we give the proof of the main result.

Proof of Theorem 1. Follows from Theorems 3.7 and 3.8 and Lemma 4.5.

We use the same notation as in the previous section. Our results apply also to establish the spectral properties of the $\bar{\partial}$-canonical solution operator. This is the densely defined operator $S$ from $\mathcal{A}^{2}\left(\mu_{m}\right)$ into $L^{2}\left(\mu_{m}\right)$ defined on Laurent polynomials $g$ by $S(g)=u$ where is the solution to the $\bar{\partial}$-equation

$$
\bar{\partial} u=g
$$

and $u$ is orthogonal to holomorphic functions in $L^{2}\left(\mu_{m}\right)$. Then, using the reproducing property of the Bergman kernel, a little computing shows that the canonical solution operator can be expressed as

$$
S g=H_{\bar{z}}(g)
$$


Therefore, by our results, the operator $S$ extends boundedly from $\mathcal{A}^{2}\left(\mu_{m}\right)$ into $L^{2}\left(\mu_{m}\right)$ as long as $m \geq 1$ and compactly as long as $m>1$.

Acknowledgement. The last author was supported by the University project of Mohamed V University and by URAC 03.

\section{REFERENCES}

1. P. Ahern, E.H. Youssfi and K. Zhu, Compactness of Hankel operators on Hardy-Sobolev spaces of the polydisk, J. Operator Theory 61 (2009), 301-312

2. G. E. Andrews, R. Askey and R. Roy, Special functions, Encyclopedia of mathematics and applications, Cambridge university press. 2001.

3. J. Arazy, S.D. Fisher and J. Peetre, Hankel operators on weighted Bergman spaces, Amer. J. Math. 110 (1988), 989-1053.

4. M. Aslam Chaudhry and S.M. Zubair, On class of incomplete Gamma functions with applications, Chapman and Hall CRC. 2001.

5. W. Bauer, Mean oscillation and Hankel operators on the Segal-Bargmann space, Integral Equations Operator Theory 52 (2005), 1-15.

6. C.A. Berger, L. Coburn and K. Zhu, Toeplitz Operators and Function Theory in nDimensions, Lecture Notes in Math., 1256, Springer, 1987.

7. H. Bommier-Hato and E.H. Youssfi, Hankel operators on weighted Fock spaces, Integral Equations Operator Theory 59 (2007), 1-17.

8. H. Bommier-Hato and E.H. Youssfi, Hankel operators and Stieltjes moment problem, J. Funct. Anal. 258 (2010), 978-998.

9. A. Bultheel, C. Diaz-Mendoza, P. Gonzalez-Vera and R. Orive, On the convergence of certain Gauss type quadrature formulas for unbounded intervals, Mathematics of computation, 69, 721-747.

10. L. Cochran and S. Clement Cooper, Orthogonal Laurent polynomials on the real line, in : Continued fractions and orthogonal functions (Loen, 1992) eds. S. Clement Cooper and W. J. Thron, Lecture notes in pure and appl.math, Dekker, N. Y, 154 1994, 47-100.

11. E.T. Copson, Asymptotic expansions, Combridge tracts in mathematics ans mathematical physics. 1965.

12. C. Diaz-Mendoza, P. Gonzalez-Vera and M. Jiménez-Paiz, Strong stieltjes distributions and orthogonal Laurent polynomials with applications to quadratures and padé approximants, Math. Comp. 74, 1843-1870.

13. A. L. Duran and R. Estrada, Strong moment problems for rapidly decreasing smooth functions, Proc. Amer. Math. Soc. 120 (1994), 529-534.

14. A. Erdélyi, W. Magnus, F. Oberhettinger and F. Tricomi, Higher Transcendental Functions, Vol. II, McGraw-Hill, New York, 1953.

15. F. Haslinger, The canonical solution operator to $\bar{\partial}$ restricted to Bergman spaces and spaces of entire functions, Ann. Fac. Sci. Toulouse Math. 11 (2002), 57-70.

16. F. Haslinger and B. Helfer, Compactness of the solution operator to $\bar{\partial}$ in weighted $L^{2}$ -spaces, J. Funct. Anal. 243 (2007), 679-697.

17. F. Haslinger and B. Lamel, Spectral properties of the canonical solution operator to $\bar{\partial}$, J. Funct. Anal. 255 (2008), 13-24.

18. E. Hendriksen, A characterization of classical orthogonal Laurent polynomials, Nederl. Akad. Wetensch. Indag. Math. 50 (1988), no. 2, 165-180.

19. W.B. Jones, W. J. Thron and H. Waadeland, A strong stieltjes moment problem, Trans. Amer. Math. Soc. 261 (1980), 503-528.

20. W.B. Jones and W.J. Thron, Orthogonal Laurent polynomials and Gaussian quadrature, in : Quantum Mechanics in Mathematics, Chemistry and Physics, eds. K.e E. Gustafson and W. P. Reinhardt, Plenum Press, New York, (1981), 449-455. 
21. W. Knirsch and G. Schneider, Continuity and Schatten-von Neumann p-class membership of Hankel operators with antiholomorphic symbols on (generalized) Fock spaces, J. Math. Anal. Appl. 320 (2006), 403-414.

22. G.L. Lopes, On the convergence of padé approximants for stieltjes type functions, Math. USSR-Sb. 39 (1981), 281-288.

23. S. Lovera and E.H. Youssfi, Spectral properties of the $\bar{\partial}$-canonical solution operator, J. Funct. Anal. 208 (2004), 360-376.

24. J.D. Murray, Asymptotic analysis, Second edition. Applied Mathematical Sciences, 48. Springer-Verlag, New York, 1984.

25. R. Meise and D. Vogt, Introduction to Functional Analysis, Clarendon Press, Oxford, 1997.

26. O. Njastad and W. J. Thron, The theory of sequences of orthogonal L-polynomials, Det Kong. Norske Vid. Selsk. 1 (1983), 54-91.

27. G. Schneider, Hankel operators with antiholomorphic symbols on the Fock space, Proc. Amer. Math. Soc. 132 (2004), 2399-2409.

28. K. Seip and E.H. Youssfi, Hankel operators on Fock spaces and related Bergman kernel estimates, J. Geom. Anal. 23 (2013), no. 1, 170-201.

29. K. Stroethoff, Hankel operators in the Fock space, Michigan Math. J. 39 (1992), 3-16.

30. N.M. Temme, An introduction to the classical Functions of mathematical physics, Library of Congress Cataloging in Publication Data.

31. Z.X. Wang and D.R. Guo, Special functions, World scientific publishing, Co. Pte. Ltd.

32. G.N. Watson, A treatise on the theory of Bessel functions, Combridge at the university press. 1944.

33. E.T. Whittaker and G.N. Watson, A course of modern analysis (An introduction to the general theory of infinite processe of analytic functions with an accunt of the principal transcendental functions), Comridge university press. 1996.

34. J. Xia and D. Zheng, Standard deviation and Schatten class Hankel operators on the Segal-Bargmann space, Indiana Univ. Math. J. 53 (2004), 1381-1399.

35. K. Zhu, Hilbert-Schmidt Hankel operators on the Bergman space, Proc. Amer. Math. Soc. 109 (1990), 721-730.

36. K. Zhu, Operator Theory in Function Spaces, Marcel Dekker, New York, 1990.

37. K. Zhu, Schatten class Hankel operators on the Bergman space of the unit ball, Amer. J. Math. 113 (1991), 147-167.

1 Faculté des Sciences de Rabat, Université Mohamed V, B.P. 1014, Rabat, MorocCo.

E-mail address: zerouali@fsr.ac.ma

2 Aix-Marseille Université, CNRS, Centrale Marseille, I2M, UMR CNRS 7373, CMi, 39 Rue F-Juliot-Curie 13453, Marseille Cedex 13, France.

E-mail address: alhomsi@cmi.univ-mrs.fr; el-hassan.youssfi@univ-amu.fr;

hachadi@cmi.univ-mrs.fr 